\title{
PLS-Based Robust Inferential Control for a Packed-Bed Reactor
}

\author{
H.M. Budman, T. Holcomb and M. Morari* \\ Chemical Engineering 210-41 \\ California Institute of Technology \\ Pasadena CA 91125
}

\begin{abstract}
Abetract
This paper compares the performance of two different inferential achernes when applied to an experimental packed-bed reactor. The first acheme, proposed initially by Brocilow, is designed based on Kalman flter extimation. The second les traditional desiga uses an extimalor computed from the Partial Leat Squares regremion method (PLS). The second approach was fonsd to give superior performance when the nonlinear syotem ander study is operated in $\bullet$ wide range of operating points. Dee to the moalinearity of the system it i csential to addres the isose of robustmess of the propoed schemes. This is formally dome in this work using Structured Singular Value Theory. For the robust nes analysis it is crucial to develop a realiztic but not overly coneerntive uncertainty description. Since the PLS estimator nes a large number of mensurements, a robust dexign based on the ancertainty aseociated with each one of the messurements would be very conservative. To overcone thi problem a lumped uncertainty description is proposed which is identified directly from experiments.
\end{abstract}

\section{Introduction}

In many industrial processes the variables to be controlled cannot be measured fast enough to achieve desired control objectives or, in some cases, cannot be measured at all. However, readily available process measurements such as temperatures, pressures, and flowrates contain much process information. Exploiting relationships between these secondary variables and the variables to be controlled (primary variables) holds the potential to make more demanding control objectives accessible. For instance inferring the primary variables from faster secondary measurements will improve the performance of the closed loop gystem. This investigation focuses on the application of robust inferential control schemes to a packed bed methanation reactor.

The design of an inferential scheme consists of two parts: the design of the estimator and the selection of a controller. In the present work two different approaches to this design problem were compared. The first approach, which follows the Kalman Filter type of design, assumes that the system can be modeled by a linear superposition of the effects of disturbances and ma nipulated variables on the primary and the secondary variables. Since this type of scheme was initially proposed by Brosilow [1] it will be referred to in this paper as "Brosilow-type inferential scheme".

The second approach is based on the method of Partial Least Squares regression, hence, will be described in this work as the "PLS scheme". In the PLS scheme, in contrast with Brosilow's scheme, no explicit superposition of the effects of disturbances and manipulated variables on the primary and secondary variables is assumed. Based on the results obtained using these two approaches, two main issues will be addressed:

- Accuracy of the inferential estimator. The goal of the inferential es timator is to accurately estimate the controlled variables. It will be shown that due to the high nonlinearity of the process under study, the PLS technique results in significant performance improvement compared to the Brosilow approach.

- Robustness of the inferential scheme. Due to the nonlinearity of the process and the wide range of operating conditions considered, studying the robustness of the proposed schemes, i.e. their operability in the presence of model uncertainty, is esential. In this work the robustness issues are formally investigated in the framework of Structured Singular Value (SSV) Theory [3]. The SSV framework assumes the uncertainty to be represented by norm bounded perturbations to the transfer functions which model the process. Previously, work in the Kalman Filter iramework used additive Gaussian noise in the system differential equations to describe the uncertainty, a less realistic description of model uncertainty. To our knowledge this work is the first experimental application of SSV theory to the design of a control system for a pilot plant chemical process, a fixed bed methanation reactor. Using this theory, it will be shown that a key issue for the analysis is the selection of an appropriate representation of the uncertainty in the system. By appropriate representation we mean realistic but not overly conservative.

In the present application, a packed bed methanation reactor is operated under conditions of partial conversion of reactants. This experimental system is deseribed in full detail in [16].

The reaction studied is the methanation of $\mathrm{CO}_{2}$ :

$$
\mathrm{CO}_{2}+4 \mathrm{H}_{2} \rightarrow \mathrm{CH}_{4}+\mathrm{H}_{2} \mathrm{O}
$$

The problem is to control the maximal bed temperature and the exit concentration of the reactant by manipulating the recycle flowrate and the heater power at the inlet to the reactor. The variables have to be controlled in the presence of changes in the reactor wall temperature. The control objectives are to obtain stability and good performance over a range of different operating conditions.

Several investigations were conducted on the issue of inferential control using Kalman Filter techniques $[1,5,8,9]$. Special emphasis was given in these works to the issue of measurement selection. These investigations can be grouped into two main categories according to how model uncertainty is accounted for in the analysis.

In the first approach, $[5,8,9]$ the system is modeled using the partial differential equations of heat and mass balance. The second approach to the measurement selection problem describes model uncertainty as norm bounded perturbations to the transfer functions describing the system $[1,11]$. This is in contrast to the previous approach in which uncertainty was represented by the process noise. This norm bounded nncertainty is relatively easy to quantify from experimental data [17] and permits one to incorporate in a clear manner the issues of robustness [14]. This type of uncertainty representation was initially introduced, in the context of measurement selection, by Brosilon [1]. The application of this technique to the fixed reactor was presented in detail in a previous works $[17,18]$.

As mentioned above, the objective is to design an inferential scheme which will result in good performance in a range of different operating conditions. The system under study, the fixed bed reactor, is known to exhibit highly nonlinear behavior in the selected window of operation. In all the aforementioned studies the estimator is designed based on data obtained from a linearization of the system around a preselected nominal operating condition. Therefore, inaccurate estimation is expected when the system is operated "far" from this nominal condition.

Partial Least Square (PLS) uses a different approach to deal with the nonlinearity of the proces. PLS was originally proposed by analytical chemists for "multivariable calibration problems" [7] and applied lately by Mejdell et al. [13 to a diatillation column for estimation of composition. Via the PLS approsech a linear relation between the primary and both the secondary and the manipulated variables over a wide range of operating conditions is established. This has two direct benefits. First, this technique requires less strong assumptions than the Kalman Filter technique regarding the linearity of the process. Use of the Kalman Filter method assumes that the effects of disturbances and manipulated variables on both the primary and secondary variables obey superposition. In the PLS approach these assumptions are not required.

Second, ance the estimator is based on a range of operating conditions, changing from one operating condition to another does not require the development of a new eatimator; from the outset, the need to function under different conditions is addressed. A main drawback of the PLS technique is that in its current form only static estimation is performed and it is not obvious how to extend the method to deal with dynamic information. In this work the dynamics of the estimator is corrected by augmenting the static estimator with a lead-lag compensator.

Using the PLS technique the accuracy of the estimator tends to increase as the number of measurements is increased. According to Brosilow increasing the number of sensors may result in increasing sensitivity to model error. 
However, it will be abown that this increased sensitivity is an artifact of the assumed uncertainty structure in the systern.

In the present work we adopt the poliey that a fixed number of measurements is available at fired position along the axis of the reactor and we are not free to either increase their number or change their positions. Therefore the rensors to be used for eatimation with either one of the eatimators discumed above have to be sefected from this et of fixed sensors.

We considered disturbances in the reactor wall temperature only. It will be shown that the steady atate bed temperatures and the exit concentration change significantly with small changes in the wall temperature.

\section{Brosilow's Inferential Control}

The Brosilow inferential control scheme was developed for systems that can be deacribed by:

$$
\begin{aligned}
& =G_{e d d}+G_{a m} m \\
& c=G_{e d d}+G_{e m} m
\end{aligned}
$$

where
- is the veetor of secondary measurements
c is the vector of controlled variables,
$m$ is the vector of manipulated variables,
$d$ is the vector of unmeasured disturbances, and
$G_{i j}$ is the transfer funetion relating output vector $i$ to input vector

In principle, all possible combinations of temperature (secondary) measurements should be considered when selecting the measurement set for inference. In the investigation of the Brosilow method, we limit the search to a single "best measurement." Beyond the obvions motivation to keep the estimator as simple as possible, additional arguments are:

- Changes in reactor wall temperature are the only type of disturbance considered in the experiments. If the sensor noise is negligible, it is posible to obtain accurate timates of a single disturbance using one single measurement. Intuitively, one will expect that for a nonlinear system such as the one under study an increasing number of measurements may improve the estimation accuracy. It can be shown that this is not the case for a Brosilow-type estimator which uses a large number of sensors spplied to a nonlinear process [2].

- We observed that as more measurements are used for estimation it be comes more difficult to satisfy the robust stability criterion. To illustrate this point we investigated the case where two secondary variables are considered for estimation. We examined 12 different pairings, each one including the maximal bed temperature and one of the 12 measure ments along the reactor. A neceseary condition condition for robust stability, based on steady state conditions, was developed in [2]. None of the candidate pairs considered for estimation satisfied this condition.

Following these considerations, one single sensor is used to infer the effect of the external disturbance on both controlled variables: maximal bed temperature and exit concentration.

Based on the method of Lee and Morari [11] the "best sensor" was found to be the one elocest to the hotspot but never crossed by it. This thermocouple is positioned at approximately 5 inches away from the reactor exit.

The controller was designed based on the IMC factorization of Holt and Morari [6]. This factorisation procedure leads to a dynamically decoupled gystem with the minimal possible delays in the controlled variables. The IMC robustnes filter time constant is found from robust stability requirement. Based on a Structured Singular Value test this time constant was found to be 83 seconds. The controller design was presented in full details in [18].

Summarixing, two main deficiescies were observed when using the previous scheme for the reactor:

- Inaccurate estimation

\section{- High sensitivity to model uncertainty}

The objective of the estimator is to estimate the primary variables as accurately as possible, i.e the exit reactant concentration and the maxima bed temperature. This objective has to be achieved for the nonlinear system when operated in a range of different operating conditions. To partially test if this objective is achieved when using the estimator proposed above, the reactor was operated in open loop around eighteen different operating points in the selected window of operation. At each one of the operating points the temperature values obtained with the 12 thermocouples and the gas chromatographic readings of the concentration were recorded when the system reached steady state.
We tested the accuracy of Brosilow's estimator which uses sensor 13 for estimation. This measurement was found in the previous section to give the best steady state performance. For simplicity we conducted the comparioon for the exit reactant concentration only. Using the temperature values st thermocouple 13 and the correspondent values of the manipulated variables, the Brosito inferential eatimator for concentration is:

$$
y=\left(G_{e m}-G_{e d} G_{e d}^{-1} G_{e m}\right) \delta m+G_{e d} G_{e d}^{-1} \delta T+y_{n}
$$

where

$\delta m$ is the deviation in menipulated variables, $\delta T$ is the temperature deviation at sensor 13 , and $\eta_{n}$ is the concentration at the nominal operating condition.

\begin{tabular}{|c|c|c|c|c|}
\hline Type of error & $\begin{array}{c}\text { Brosilow } \\
1 \text { sensor } \\
(\% \text { vol })\end{array}$ & $\begin{array}{c}\text { Brosilow } \\
12 \text { sensors } \\
(\% \text { vol })\end{array}$ & $\begin{array}{c}\text { PLS } \\
1 \text { sensor } \\
(\% \text { vol })\end{array}$ & $\begin{array}{c}\text { PLS } \\
(\% \text { vonsors })\end{array}$ \\
\hline \hline worst error & 0.65 & 0.77 & 0.19 & 0.06 \\
\hline RMS error & 0.20 & 0.23 & 0.08 & 0.02 \\
\hline
\end{tabular}

Table 1: Steady state experiments using Brosilow's and PLS estimators

The development of this estimator is given in [2]. From the results shown in Table 1 we observe that significant errors up to $0.65 \%$ ool of $\mathrm{CO}_{2}$ occurred. We observe that the differences between the estimates and the actual values tend to increase as the system is operated "far" from the selected nominal condition which corresponds to 17 slpm recycle flowrate and $30 \%$ heating power.

As mentioned in the Introduction, Brosilow concluded that for his choice of sensor noise and process noise respectively, a larger number of measurements will result in more accurate estimation. It is interesting to test whether this conclusion still applies for the reactor operated at different operating points. For this purpose we compute a static estimator which uses all available 12 temperatures along the reactor.

Table 1 compares gas chromatographic readings for the different conditions with concentration estimates computed from (3). From the results we can conclude that overall the accuracy decreased; both the RMS and worst errors increased as compared to the one measurement estimation case. However, certain improvements were observed for points "close" to the nominal operating point. Since Brosilow's estimator was designed for linear systems with additive Gaussian process and sensor noise, the pattern of improvement "cloee" to one operating point coupled with performance deterioration "far away" should not be surprising. The effect of noise terms on the estimate can be expected to be filtered by this estimator as more measurements are used. However as we demonstrated, with more measurements the estimator will not necessarily be more accurate when applied to a nonlinear system operated at different conditions. It has to be emphasized that the Brosilow estimator in effect assumes linear superposition of the effects of disturbances and manipulated variables on primary and secondary variables. This assumption can be arbitrarily inaccurate for nonlinear systems as demonstrated by the results in this section. This shortcoming is the motivation to look for an alternative estimator which will be more accurate for the nonlinear process studied in the present work.

In the Brosilow inferential scheme the controller is the inverse of the plant $G_{c m}$ at steady state. The condition number of $G_{\mathrm{cm}}$ was found to be 812 . This large condition number is due to the fact that the controlled variables are highly correlated at least in a part of the selected window of operation. This fact was found both experimentally in the present work and through numerical simulations in a previous work by Mandler[12]. In general this large condition number results in high sensitivity to model uncertainty[14]. This explains partially why the robust stability condition could not be satisfied even for a small number of measurements. An additional difficulty resulting from the high condition number is that the manipulated variables are easily driven to their saturation limits for relatively small external disturbances. For example, the heating power reaches its saturation limits for a change of the order of 3 degrees in the wall temperature. Therefore an alternative dynamic compensator will be designed to avoid the aforementioned problems. This compensator will be used in conjunction with a Partial Least Squares estimator. The design of the compensator and the PLS estimator are presented in the next section.

\section{Partial Least Squares Estimation}

In this section we will present the second scheme for inferential control for which the eatimator is computed using the Partial Least Squares regression technique. We will also address the issues of robustness related to this type of scherne. 


\subsection{PLS Estimator}

The PLS design procedure secks to minimize the squared error between measurements of primary rariable and predietions of these primary variables made from secondary measurements for a set of different operating conditions. For this application, the primary variables were outlet $\mathrm{CO}_{2}$ concentration, and the secondary messurements were both manipulated variables and all twelve temperature measurements along the reactor. One should note that because only steady state dats are considered, a static estimator is obtained. We found that a simple parabolic interpolation of the three highest contiguous temperature measurements along the reactor yielded good estimates of the maximal temperature. Therefore, the PLS regreasion will be used solely to estimate the outlet concentration while the parabolic interpolation will be ved to eatimate the temperature. In general we expect that the sccuracy of the regresion will improve for an increasing number of measurements, hence, all 12 fixed thermocouples will be used for estimation.

In the PLS technique the regresion is performed direetly from manipulated rariables and temperature measurements to the concentration. This is perhape the main rewon that the PLS extimator is expected to produce more accurate eatimates then Brocilow's estimator. For Brosilon's and Kalman Filter sebemes the transfer functions $G_{o m}, G_{o d}, G_{c e}$ and $G_{o m}$ are separately identified and then the concentration is computed from (3) or a variant of (3) in the care of the Kalman filter. Thus, the coefficients for $\delta m$ and $\delta T$ in the estimator are asembled from algebraic operations of the separately identified trenefer functions. This in in clear contrast with the PLS eatimator where these coefficiente are computed directly from the regreasion procedure. The improvement in estimation accuracy resulting from this procedure will be clearly illuteated in this seetion.

Before progrensing, a review of the PLS regression algorithm is in order. The PLS algorithm was developed by Wold [15]. An excellent tutorial has been provided by Geledi and Kowalski [4], while Höskuldsoon [7] bras done - thorough job of analyzing the mathematical aspects of the algorithm. A weakneses of PLS in that one bas to decide how many directions to use, and that arbitrary scale transformations will alter the outcome of the re gression. At present there is no theoretically general and accepted method for determining the number of directions to use.

For the reactor date, fourteen deta points representing fourteen different steady state operating conditions were available. Each data point consists of twrelve temperature meavarenients and 2 manipulated variables. Thus, the problem would appear to have an exact solution. However, the input data are highly correlated, making the problem very ill-conditioned. To addres this problem, the PLS approach was applied. The two manipulated variables were scaled to have unity variance, and all temperstures had the same scaling factor applied; this angle scaling factor was chosen so that the average variance of the scaled temperatures was unity, and seven directions were used. Further details on the development of the PLS estimator are in [2].

The accuracy of this PLS estimator is tested once again using the steady state data presented in Table 1 . The results of the estimation for the different operating point are shown in the same table. The worst error between the PLS extimation and the actual concentration is $0.06 \%$ vol in $\mathrm{CO}_{2}$ concentration. This a aignificant improvement in the accuracy as compared to the Brosilon type extimator. We also tested the conjecture that the accuracy of the PLS estimator may increase with an increase in the number of messurements. For comparimon we compute a PLS estimator which uses one single temperature for estimation and compare it to the estimates obtained with the 12 sensor based estimator. Serisor 13 was selected since this is the sensor selected for Brosilow's extimator. The estimator is tested for all 18 operating points. The worst error obtained is $0.19 \% \mathrm{CO}_{2}$ concentration, i.e. 3 timies larger than the work error obtained when all 12 temperature measurements were used for estimation.

The main drawback of the PLS estimator is that it is based on steady state information. Therefore, it is expected to produce good estimates at steady state but will be inaccurate for the dynamic situation. The formal extension of the PLS technique to deal with dynamic information is an open field of research. To test the dynamic accuracy of the estimator the time response of the actual exit concentration to - step in recycle flowrate is measured (see Figure 1). The time constant for this response is of the order of 1200 seconds. If the concentration computed with the PLS static estimator is examined for this experiment, a time constant of approximately 300 seconds is obeerved (see Figure 1). The chearly indicates that the estimator dynamics obtained from the steady state data is inaccurate.

To correct this situation we propose to augment the PLS estimator with a simple lead-lag compensator:

$$
\delta y_{0}=\frac{1+\tau_{A} \delta}{1+\tau_{B} \delta} \delta y_{0}^{P L S}
$$

The sero and pote of this compensator $\tau_{A}$ and $\tau_{B}$ were obtained by fitting them to the actual data from the step response to recycle flowrate shown in Figure 1 around the nominal operating condition(17 slpm recycle-30\% beating power). The time response for the concentration using the estimator given by (4) is shown in Figure 1.

The estimator given by (4) hes identical structure to the suboptimal extimator propoced by Brocilom an alternative to the relatively complex optimal Kalmas filter. Brovilon applied his inferential control to a distillation process. For this process he compared the closed loop performance using the optimal Kalman filter to the performance obtained using a suboptimal estimator finding minimal differences between the two approaches.

\subsection{Uncertainty Description}

The PLS estimator proposed in this section uses a large number of measurements for estimation. This raises the question of an appropriate uncertainty description. Brosilow's analysis asoumes that uncertainty is associated with each one of the measurements. Uaing this uncertainty description in the PLS acheme would be extremely conservative for the analysis, since the uncertainty regions for all the measurements would be added together. The worst panible combination of all there individual uncertainties would then define the uncertainty region for the predictar. Since the PLS scheme assumes that correlations between the necondary variables exist, such an uncertainty description would be inberently conservative and would become more conservative as more memurements are considered. Therefore, an alternative uncertainty description is proposed which lumps together the individual uncertainty bounds into a more compact structure.

The bey insight leading to the lumped uncertainty description is to realize that the secondary measurements are only a means to an end. Uncertainties in these variables are only important in as much as they affect the overall accuracy of the estimator. Thus, only the uncertainty of the estimation is considered; the identification of the iridividual uncertainties is completely bypased. The first step is to identify a transfer function which relates the manipulated wariables directly to the estimated controlled variables. To identify this tranefer funetion, the PLS estimator for concentration and the parabolic interpolation algorithm for the maximal temperature were implemented on the experimental system. Subsequently linear transfer functions relating the estimated controlled variables to the 2 manipulated variables and the disturbance are directly identified from step experiments. The resulting block diagram is shomn in Figure 2. The actual controlled variables are represented by $c$ and the estimated variables by $\hat{c}$. To simplify the analysis we assume:

$$
\hat{G}_{c m}=\hat{G}_{c m}
$$

\begin{tabular}{|c|c|c|c|}
\hline & Gain & Time constant(sec) & $\overline{\text { Delay}(\sec )}$ \\
\hline$\overline{\mathbf{G}_{Y H}}$ & $-0.007--0.015$ & $1800-2200$ & $0-160$ \\
\hline $\mathbf{G}_{Y R}$ & $0.06-0.16$ & $1193-1507$ & 0 \\
\hline $\mathrm{G}_{T_{\text {mex }}}$ & $0.12-0.3$ & $500-1000$ & $625-750$ \\
\hline $\mathrm{G}_{T_{\text {mes }} R}$ & $-3.0--2.75$ & $245-255$ & 0 \\
\hline
\end{tabular}

Table 2: Uncertainty bounds for gains,time constants and delays for the transfer function relating manipulated variables to the estimated concentration uning PLS and the interpolated maximal bed temperature.

The procedure for identifying the nominal transfer functions and the respective uncertainty elements is the same as the one used in our previous work [17]. The main idea is to model the system through step response experiments around different operating conditions in the window of operation. As before, it is asomed that the system can be approximated by a first order system with a delay. The nominal models for $G_{i m}$ and the variation in the time constants and delays along the window of operation are identified from step responses arousd different operating points(see Table 2): This parameter variation can be translated using the technique of Laughlin [10] to an uncertainty description suitable for robustness analysis.

The uncertainty structure in Figure 2 is defined by:

$$
G_{i m}=\hat{G}_{i m}+\Delta_{i m}^{*}
$$

where

$$
\begin{aligned}
& \hat{G}_{t_{m}}=\left(\begin{array}{cc}
\hat{G}_{Y R} & \hat{G}_{Y B} \\
\hat{G}_{T_{\max } R} & \hat{G}_{T_{\max }}
\end{array}\right) \\
& \Delta_{i m}^{*}=W_{e_{2}} \Delta_{i m} W_{e_{1}} \\
& W_{e_{1}}=\left(\begin{array}{cc}
W_{Y R} & 0 \\
W_{T_{\text {max } R}} & 0 \\
0 & W_{Y H} \\
0 & W_{T_{\text {mos }}}
\end{array}\right)
\end{aligned}
$$




$$
\begin{aligned}
w_{e_{1}} & =\left(\begin{array}{llll}
1 & 0 & 1 & 0 \\
0 & 1 & 0 & 1
\end{array}\right) \\
\Delta_{i_{m}} & =\quad \operatorname{diag}\left(\Delta_{1}, \Delta_{2}, \Delta_{3}, \Delta_{4}\right) \\
\bar{\sigma}\left(\Delta_{i}\right) \leq 1 \quad i=1,4 &
\end{aligned}
$$

where $T_{\text {max }}$ is the maximal bed temperature and $Y$ is the exit concentration.

The aetual value of the maximal temperature is identical to its estimate aince these quantities ane obtained using the same parabolic interpolation with three temperature measurements. Therefore $c$ differs from $\hat{c}$ only in the value of the concentration. The estimated value is obtained with the PLS regreasion while the real value is mearured using the gas chromatograph. For the purpose of the saalysis, an additive noine term $\mathrm{m}$ is added to the real concentration to account for the difference between the estimated and the real concentration due to regremion errors. The magnitude of this noise is selected to be appracimately equal to the worst error between eatimates and real values according to the results in Table 1 , i.e. $\pm 0.06 \% \mathrm{CO}_{2}$ concentration.

\subsection{Design of the dynamic compensator}

Using the inverse proces model as controller shown to cause stability problems [2]. These problems are due to the large condition number of the process trander function. In order to correct this situation a pragmatic decision is made regarding the performance requirements for the control scheme. Accurate control of the concentration requines integral action. Bowever, if one oaly wistes the temperature to be maintained at a reasonable level to avoid danage of the catalyat, then offet can be tolerated in this controlled variable. The concentration is paired to the recycle flow, and the maximal temperature is paired to the heating power. Since the concentration reacts much faster to recycle flowrate than to heating power the above pairing is expected to give better performance for control of concentration than the reverse pairing.

Explicit conditions were developed for the pairing problem for the case where integral action is required for all the loops[14]. Since integral action is not required in our case for the maximal temperature these conditions do not apply to our problem. A more formal approach to the pairing selection is left for future investigations. A simple diagonal controller is proposed with proportionalinfegral control for the concentration and only proportional control for the maximal bed temperature:

$$
C=\left(\begin{array}{cc}
K_{1}\left(1+\frac{1}{r_{16}}\right) & 0 \\
0 & K_{2}
\end{array}\right)
$$

The expected offset between measured and predicted concentration, expremed by the noise $\mathrm{n}$, is due to the regression error and is not affected by the selection of the controller parameters. On the other hand, the controller parameters can be selected to minimize the expected offset in the maximal temperature. The procedure used by Lee and Morari [11] for measurement selection is und in this cane for selection of the controller parameters. As in that case for the analyeis the controlled variables $c$ and the disturbance $d$ are multiplied by a performance weight $W_{p}$ and a disturbance weight $W_{d}$ respectively.

The expected range of variation for the maximal temperature was to be $\pm 15^{\circ} \mathrm{C}$ [2]. Then in order to scale the maximal temperature to one and to obtain integral action in concentration the performance weight $W_{p}$ is selected as:

$$
W_{p}=\left(\begin{array}{cc}
\infty & 0 \\
0 & \frac{1}{15}
\end{array}\right)
$$

For disturbances of $\pm 2{ }^{\circ} \mathrm{C}$ the disturbances weight is $W_{d}=2$.

The bloct diagram with the weighte in shown in Figure 2. This block diagram can transformed into the $M-\Delta$ structure. Using this structure the controller parameters can be computed from a two step iterative proceduse as described in [2].

In the present wort we compute the PLS estimator using all the measurements available along the resctor. We assumed that their location cannot be changed and their number cannot be increased. For this situation we concluded based on the ateady state results that the estimator which uses all arilable measurements is most accurate.

\section{Closed-Loop Experiments}

The main goal of the clooed loop experiments is to test the stability and performance of the two inferential control schemes used in this work. By comparing the theoretical predictions of stability and performance to the experimental results, it will be possible to verify the identified uncertainty of the process. The stability and the performance results are presented separately in the sequel for each one of the schemes.

\subsection{Experiments for Brosilow's scheme}

The experiments described betow are conducted wing thermocouple \#13 for inference. Thus, both controlled variables, maximal temperature and exit concentration are inferred from sensor \#13.

Stability Results. As thom in [17], the stability of the closed loop depends only on the uncertainty in $G_{s}$. Therefore, it is possible to validate the uncertainty $\Delta_{, m}$ by comparing the experimeatal to the theoretical stability results. The IMC time conatant required for robust stability as computed in section 4 is $\lambda \geq 82$ mece.

In order to chect this result in the experimental system, we studied the disturbance rejection problem for different operating conditions uring different filter time conetants: $58 \mathrm{sec}_{2} 82$ sec and $140 \mathrm{sec}$. These experiments are conducted in the following manner:

-The premure in the Dowtherm container is increased resulting in a slow inerease in wall temperature.

- The controller is activated at a predefined operating point.

-The presoure inside Dowtherm conkaines is released causing a step disturbance in wall temperature.

-The manipulated and controlled variables are monitored and stability of the clowed-loop determined.

\begin{tabular}{|c|l|c|c|c|}
\hline Exp\# & $\begin{array}{l}\text { Initial Operating } \\
\text { Condition }\end{array}$ & $\begin{array}{c}\text { Robustness Filter } \\
\text { Time Constant }\end{array}$ & $\begin{array}{c}\text { Magnitude of } \\
\text { Disturbance }\end{array}$ & $\begin{array}{c}\text { Stability } \\
\text { Results }\end{array}$ \\
\hline \hline 1 & $\begin{array}{l}15 \% \text { Power } \\
13 \text { slpm Recyele } \\
256^{\circ} \mathrm{C} \text { Wall Temp. }\end{array}$ & $140 \mathrm{sec}$ & $-1^{\circ} \mathrm{C}$ & Stable \\
\hline 2 & $\begin{array}{l}15 \% \text { Power } \\
13 \text { slpm Recycle } \\
256^{\circ} \mathrm{C} \text { Wall Temp. }\end{array}$ & $82 \mathrm{sec}$ & $-1^{\circ} \mathrm{C}$ & Unstable \\
\hline 3 & $\begin{array}{l}15 \% \text { Power } \\
13 \text { slpm Recycle } \\
256^{\circ} \mathrm{C} \text { Wall Temp. }\end{array}$ & $58 \mathrm{sec}$ & $-1^{\circ} \mathrm{C}$ & Unstable \\
\hline 4 & $\begin{array}{l}15 \% \text { Pomer } \\
15 \text { slpm Recycle } \\
256^{\circ} \mathrm{C} \text { Wall Temp. }\end{array}$ & $58 \mathrm{sec}$ & $-1^{\circ} \mathrm{C}$ & Stable \\
\hline 5 & $\begin{array}{l}5 \% \text { Power } \\
17 \text { slpm Recycle } \\
257^{\circ} \mathrm{C} \text { Wall Temp. }\end{array}$ & $58 \mathrm{sec}$ & $-2^{\circ} \mathrm{C}$ & Stable \\
\hline 6 & $\begin{array}{l}15 \% \text { Power } \\
19 \text { slpm. Recycle } \\
256^{\circ} \mathrm{C} \text { Wall Temp. }\end{array}$ & $58 \mathrm{sec}$ & $-2^{\circ} \mathrm{C}$ & Stable \\
\hline
\end{tabular}

Table 3: Closed-loop inferential control experiments

The operating conditions for which the experiments are performed and the stability results are presented in Table 3 . As is apparent from experiments $3,4,5$ and 6 in Table 3 , it is more difficult to stabilize the system for bwer recycle flow rates. That is, for small recycle flowrates the system appean to be most ansitive to model mismatch. By increasing the filter time constant, we are able to maintain stability at low flow rates, but at the expense of significantly reduced speed of response (experiment 1).

The syatem uncertainty was identified for flow rates between 12 slpm to 20 slpm. However using the filter time constant computed from the robust ability condition ( $82 \times$ ), instability was obtained for an operating condition corresponding to 13 slpm recycle flowrate and $15 \%$ heating powrer (experiment 2). This is not surprising considering that during the closed loop experiment the recycle flow rate becomes leas than the minimal value of 12 slpm for which the proces we identified. This situation is clearly illustrated in Figure 3. Therefore we conclude that as long the manipulated variables during the experiment remain inside the preselected window of operation, the stability condition obtained through $\mu$ analysis is verified.

Performence Realts The time history of the controlled variables associated with Experiment 5 are presented in Figure 4. Also shown in this figure are the setpoints and the expected deviations for open-loop conditions. It is apparent from these figures that the inferential control scheme rejects the disturbance in wall temperature although it leaves significant steady state offsets in both controlled variables. For simplicity, only the steady state performance criterion given, developed in [2], is compared to the experimental results. More epecifically, we check for all the experiments:

$$
\frac{\left\|W_{p} c(0)\right\|_{2}}{\left\|W_{d}^{-1} d(0)\right\|_{2}} \leq \frac{1}{c_{p}^{*}}
$$

where $1 / c_{p}^{*}=0.42$ for thermocouple \#13. Since the inequality was satisfied for all the performed experiments, we conclude that the estimated steady state uncertainty is not invalidated. 


\subsection{Experiments using the PLS Inferential Scheme}

In this case the concentration is based on the PLS regression uning 12 sensors and the 2 manipulated variebles valuea while the maximal bed temperature is estimated using parabolic intapolation of 3 messarements.

Stability Reaults. In this case the stability of the cloned loop syatem can be uned to validate the uncertainty $\Delta_{\mathrm{im}}$ (see Figure 2). Stability is tested for the condition corresponding to experiments 4,5 and 6 in Table 3 . The syctem was found to be atable for operating conditions 5 and 6 but unstable for operating condition 4. This is due once again to the fact that for operating point 4 the recycle flowrate becomed less than the minimal recycle flowrate velected for identification of the system. Therefore as concluded before, a long as the manipulated variables remain in the preselected window of operation for which the system was identified, the stability condition obtained with $\mu$ analysia is verified and consequently the uncertainty $\Delta_{\text {in }}$ is validated.

Performance Realts. The time evolution of the controlled variables for experiment 5 in Table 3 is shown in Figure 5. A steady state offset of $0.05 \%$ $\mathrm{vol} \mathrm{CO}_{2}$ is obtained for the concentration while an offet of approximately $3^{\circ} \mathrm{C}$ is obtained for the maximal temperature. The steady state offset in concentration is reduced by a factor of 4 as compared to the result obtained with Brosilon's estimator. This is in agreement with the results where the PLS estimator was shown to be overall more accurate at steady state along the considered windon of operation [2].

The estimated concentration using PLS regression was compared for experiments 5 and 6 to the actual concentration measured during these experiments. The results are shown in Figure 6. The maximal deviation obtained between the two variables is approximately $0.06 \%$ vol $\mathrm{CO}_{2}$. This is a very good accuracy considering that the accuracy of our gas chromatographic system is approximately $\pm 0.05 \%$ vol $\mathrm{CO}_{2}$. These results partially validates the dynamic correction of the PLS estimator using lead-lag compensation.

\section{Conclusions}

Two inferential control schemes are applied to an experimental methanation reactor. The performance obtained with these schemes is compared and conclusions can be drawn from this comparison. The PLS estimator is shown to be significantly more accurate for estimating the actual concentration in a wide range of operating conditions. A main disadvantage of the PLS regreasion technique is that it is based strictly on steady state information. In this work it was shown that it is poesible to correct the estimator to account for dynamic data using a simple lead-lag compensator. The zero and pole of this compensator are determined by fitting the estimate to experimental data.

The reason that the PLS extimator is more accurate than Brosilow's is that in Brosilow's estimator the transfer functions $G_{s m}, G_{, d}, G_{\mathrm{cm}}$ and $G_{e d}$ are separately identified. Then, the regression coefficients for the temperatures and the manipulated variables in the estimator are obtained from algebraic operations on these transfer functions. In the PLS technique the coefficients are identified directly from the experimental data. Thus, the separate identification of the above mentioned transfer functions is bypased and all correlations are captured.

An additional difficulty with Brosilow's scheme arises from selecting the controller as the inverse of the process model. Due to the high condition number of the process under consideration, this type of control will be very sensitive to model uncertainty. In order to overcome this problem a decentralized controller is selected and its parameters are tuned for robustness using $\mu$ criteria. The use of this controller in conjunction with the PLS estimator results in good clowed bop performance.

It was shown that for Brosilow's inferential scheme a larger number of measurements does not necessarily result in better estimation of the concentration for different operating points. This is in contrast with the PLS regreasion scheme for which the accuracy was shown to improve as the number of sensors is increased.

Developing a realistic but not overly conservative uncertainty description is crucial for analysis and-tuning. In the PLS scheme, a lumped uncertainty

-Author to whom correspondence should be addrened: phone (818)356-4186, far (818)568-8743, e-meil mmeimc.ealtech.edu. This proceedings peper is a condensation of the the journal article submitted to Induatrial and Engineering Chemistry Research [2] between the manipulated variables and the estimated controlled variables is identified directly. A design using the uncertainty associated with each one of the measurements exparately would be very conservative.

Identifying correlations between the uncertainty elements can reduce the conivervativeness of the design. This type of correlation was found in the context of the Brosilow acheme between the elements of $\Delta_{d m}$. On the other hand the PLS acheme apparently accounted for all the correlations between the parameters and no correlations were found between the elements of the uncertainty $\Delta_{\mathrm{zm}}$.

Future research encouraged by this work is a formal extension of the Partial Least Squares technique to account for dynamic information and the application of nonlinear-regrescion techniques for estimation.

Acknowledgment Hector Budman would like to achoonledge the finoncial support of the Rothechild and Bantrell Foundations. Tyler Holcomb is the recipient of a National Science Foudation Grodute Fellowship.

\section{References}

[1] C. Browilon and B. Joseph. AIChE Journal, 24:485-509, 1978.

[2] H. Budman, C. Webb, T. Holcomb, and M. Morari. Robust inferentia control for a packed-bed reactor. submitted to Industrid and Engineering Chemistry Reasearch, 1990.

[3] J. Doyle. Analyais of feedback systems with tructured nncertainties. IEEE Proceedings Part D, 129:242-250, 1982.

[4] P. Geledi and B. Kowalaki. Partial leat equares regremion: A tutorial. Anel. Chim. Acte., 185:1-17, 1986.

[5] T. Harris, J. MacGregor, and J. Wright. Optimal sensor location with an application to a packed bed tubular reactor. AIChE Josma, 26, 1980.

[6] B. Holt and M. Morari. Design of reailient procesing plants - V The effect of deadtime on dynamic revilience. Chemical Eagineering Science, 40:1229-12s7, 1985s.

[7] A. Hoskuldeson. Partial least equares regresion methods. Jowral of Chemometrics, 2:211-228, 1988.

[8] S. Jorgensen, L. Goldschmidt, and K. Clement. A sensorlocation pro cedure for chemical procesees. Computers and Chemical Engineering, 8:195-204, 1984.

[9] S. Kumar and J. Seinfeld. Optimal location of measurements in tubular reactors. Chemical Engincering Science, 33:1507-1516, 1978.

[10] D. Laughlin, K. Jordan, and M. Morari. Internal model control and proces uncertainty: Mapping uncertainty regions for SISO controller design. Intermational Journal of Control, 44:1675-1698, 1986.

[11] J. Lee and M. Morari. Robust measurement selection. Submitted to A Etomatica, 1989.

[12] J. A. Mandler. Robuat Control Syatem Design for a Fized-Bed Catalytic Reactor. PhD thesis, California Institute of Technology, 1987.

[13] T. Mejdell and S. Skogestad. Eetimate of proces outputs from multiple secondary measurements. In Proceedings of the American Control Confereace, pages 2112-2121, 1989.

[14] M. Morari and E. Zafiriou. Rotrat Process Control. Prentice Hall, 1989.

[15] W. S., R. A., W. H., and D. W. The colinearity problem in linear regression: The partial least squares approach to seneralised inverses. SIAY J. Sci. Stat. Compet., 5(3):743-753, 1984.

[16] C. Webb. Robest Control Strategies for a Fired Bed Chemical Reactor. PhD thesis, California Inatitute of Technology, 1980

[17] C. Webb, H. Budman, and M. Morari. Identifying frequency domain uncertainty bounds for robust controller design- theory with application to a fixed-bed reactor. In Proceedings of the Americen Control Conference, pages 1528-1533, 1989.

[18] C. Webb, H. Budman, and M. Morari. Robust inferential control for a packed-bed reactor. In Proceedings of the American Control Conference, pages 575-580, 1990. 


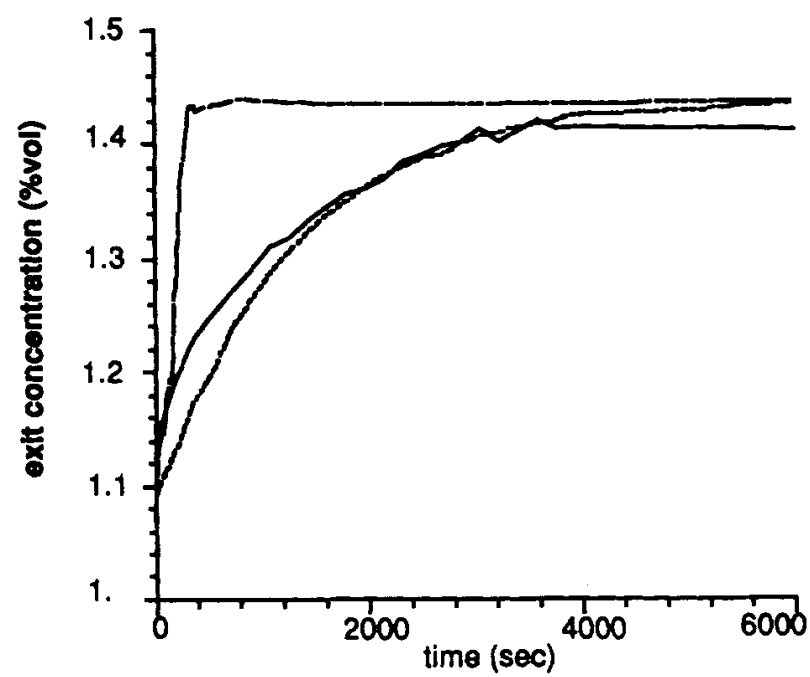

Figure 1: Time response of concentration to step in recycle flowrate for concentration: solid line-measured with GC, dot-long dash line-estimated with PLS estimator based on steady state data and dot lino-estimated with PLS estimator augmented by lead-lag compensator.

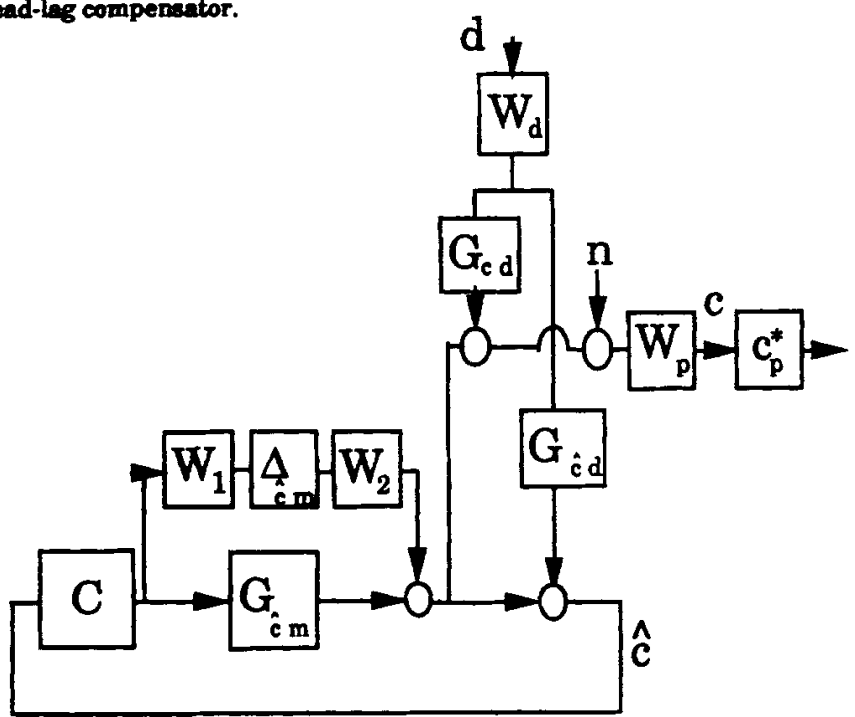

Bienre 2: Block structure for steady state performance analysis for the PLS inferential scheme.
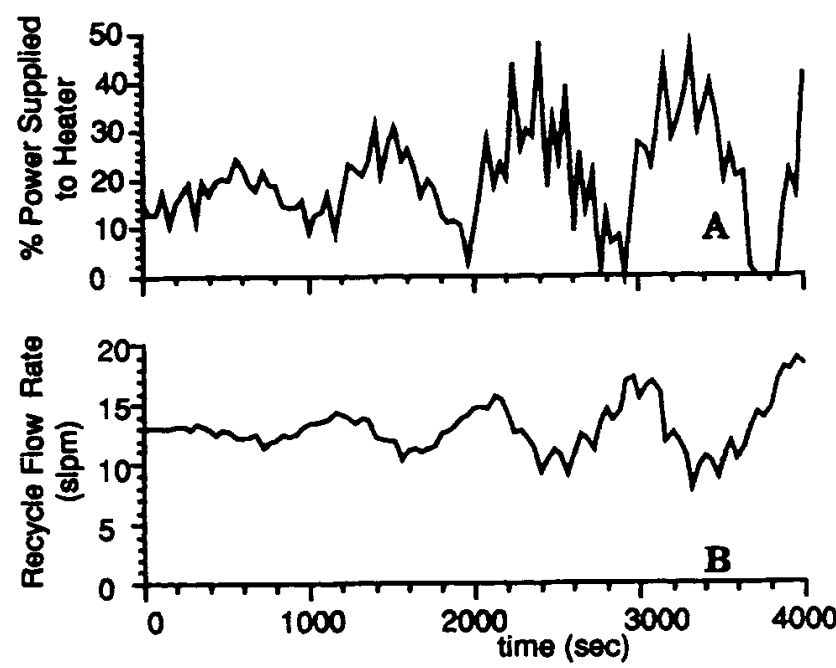

Bianre. 3: Retponse of the manipulated variables to step decreace in wall temperature (experiment 2).

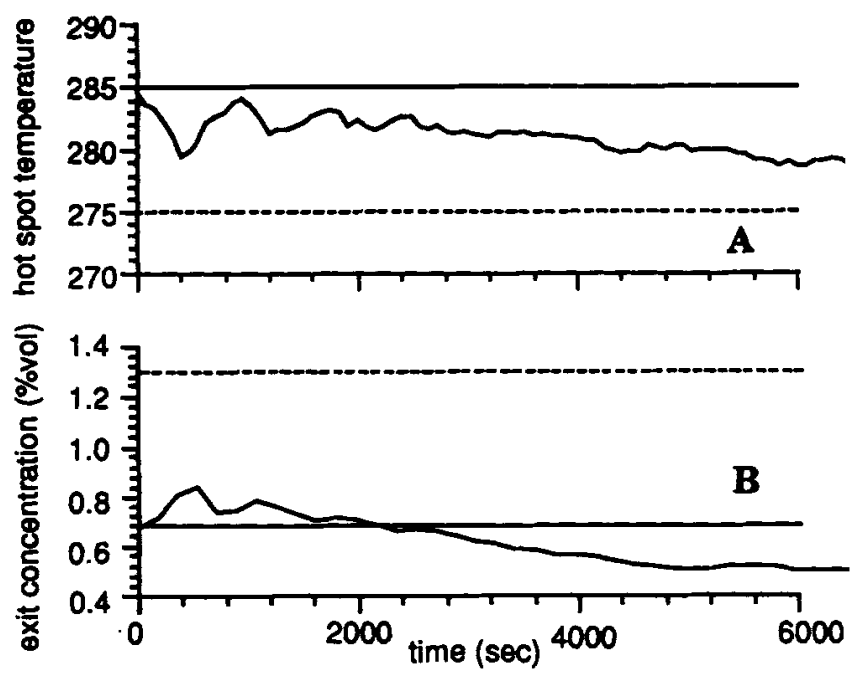

Fiaure 4: Response of controlled variables to step decrease in wall temperature (experiment 5), using Brosilow's inferential scheme: solid line-set point, dashed line-expected open loop deviation.
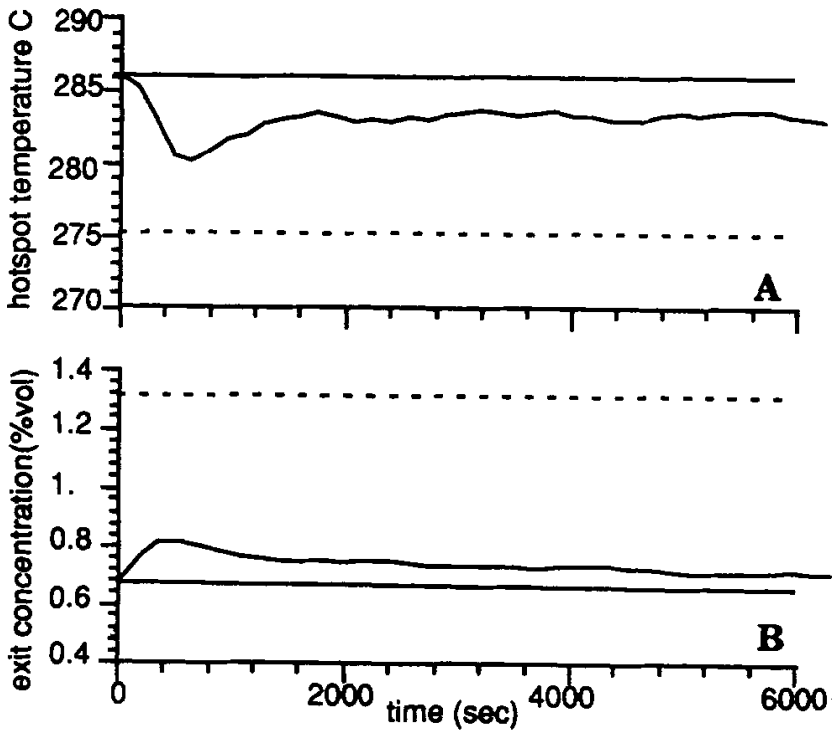

Figure 5: Response of controlled variables to step decrease in wall temperature (experiment 5), using PLS inferential scheme: solid line-set point, dashed line-expected open loop deviation.

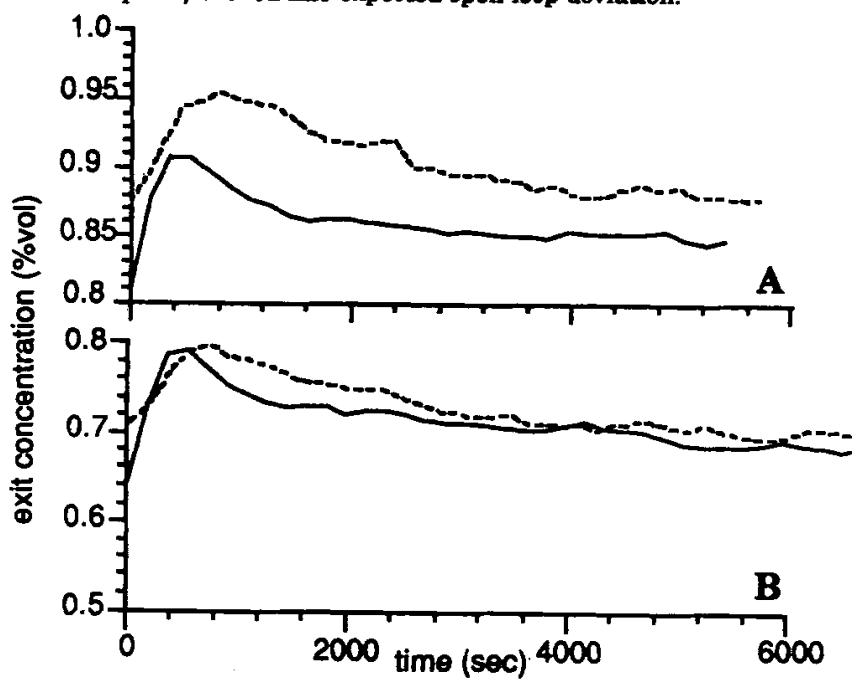

Figure 6: Comparison between exit concentration estimated by PLS estimator(dot lines) to concentration measured by GC(solid line) for a-experiment 5 , b-experiment 6 . 